\title{
Conscious Experience and Quantum Consciousness Theory: Theories, Causation, and Identity
}

\section{Mika Suojanen ${ }^{1}$}

\begin{abstract}
Generally speaking, the existence of experience is accepted, but more challenging has been to say what experience is and how it occurs. Moreover, philosophers and scholars have been talking about mind and mental activity in connection with experience as opposed to physical processes. Yet, the fact is that quantum physics has replaced classical Newtonian physics in natural sciences, but the scholars in humanities and social sciences still operate under the obsolete Newtonian model. There is already a little research in which mind and conscious experience are explained in terms of quantum theory. This article argues that experience is impossible to be both a physical and non-physical phenomenon. When discussing causality and identity as transcendental, quantum theory may imply the quantum physical nature of conscious experience, where a person associates causality to conscious experience, and, thus, the result is that the double-aspect theory and the mind/brain identity theory would be refuted.
\end{abstract}

Keywords: experience, mentality, physicality, contrariety, quantum consciousness theory, double-aspect theory, mind/brain identity theory.

\footnotetext{
${ }^{1}$ Department of Philosophy, Contemporary History and Political Science at University of Turku, Finland, email: mikasuojanenphilosophy@gmail.com
} 


\section{Introduction}

We cannot say we know what consciousness or mind is. We are only consciously experiencing. If, in the nature, all phenomena are physical, and people are also the creatures of the nature, then it follows that people's mind and psychological states are also physical phenomena. The transcendental view, especially Immanuel Kant's view, by contrast, immediately recognizes human experience from which material form cannot be directly deduced. But if everything consists of quantum particles in the nature, both living and non-living material lumps, then it is likely that experience is also a quantum phenomenon inhering in a human person, and at least within most animals. There are some logical and empirical grounds for such an assumption rather than the alternative one that experience would exist separate from and independent of the physical world, as René Descartes argued about mind. In contemporary philosophy, psychology, and cognitive science, mind-brain relations are discussed, and these relations are conceived in a direct way-mind $\leftrightarrow$ brain, where mind-matter interactions are understood in the sense of causal relations. Even though the existence of experience is generally acknowledged, eliminative materialism (Churchland 1984, 1985), for example, refuses the existence of mental entities: there is no need to discuss mental entities because all mental states and properties can be reduced to the material domain. This point of view claims that it is both necessary and sufficient to only explore and understand the material domain, e.g., the brain, in order to understand the mental domain, e.g., mind. Furthermore, the mind/brain identity theory assume that states and processes of the mind are identical to states and processes of the brain: experiences just are brain processes, not merely correlated with brain processes (Place 1956; Feigl 1958). If, on the contrary, experiences, more generally mental entities, cannot be eliminated, how should be experience understood? I examine how so-called quantum consciousness theory addresses the possibility that the material level, the brain, causes a quantum physical experience - due to causality, it cannot be reduced to the neural brain processes. Since the origin of the experience and its essence cannot be derived directly from the experience, the conclusion can be derived from the intellect and be transcendental in the Kantian way.

It is not easy to say exactly what experience is, to point to it and say, "This is experience". We are conscious and just have experiences (Strawson 2016), which implies that experience is a property belonging to someone. This leads us transcendental, however. The person is immediately aware of experiences, but certain general features of objects cannot be derived from experience, such as causality, identity, or necessity. By the concept of transcendental, Kant meant exactly that the intellect constitutes these general features to the object so that one can be aware of the object (Kant 1998, 149 (B25)). From the perspective of the first person, experience seems to appear for some reason. It occurs regardless of our actions. Thus, the concept of causation may be appropriate to experience, even though the causes of experience are not observable. In fact, experience does not necessarily reduce to states and processes of the brain. Direct relations of mind and brain may be non-reductive if the material brain is not necessary or not sufficient to explore and understand mental entities. Within this non-reductive framework, the causal influence between mental states and material states becomes relevant. Because causation and identity have different meanings, I will argue that the inclusion of causality in the theories of consciousness prevents the presence of identity — we are discussing this at the end.

In the following pages, I will show that there are rational justifications in support of the assumption that conscious experience is not identical to a brain process but exists as a physical quantum entity. First, I will shortly present reasons to believe in the mentality of experience, reasons to describe experience as mental, i.e. non-physical. In the search for the nature of 
experience, we will perceive that the existence of experiences requires some mechanism that will make them occur. Next, according to quantum consciousness theorists, it seems to be evident that philosophy and other human sciences have not passed on a change in natural sciences, when quantum physics replaced classic Newtonian mechanics. I will look for evidence for quantum physical experience from research in which consciousness and experience are explained by quantum mechanics. Finally, when the two previous sections are combined, we will perceive them incompatible. If experience is a physical quantum phenomenon, then it is not a simultaneous non-physical phenomenon, a phenomenon associated with a "Cartesian mind". Therefore, I will argue that experience is impossible to be both a physical and a non-physical phenomenon because an object cannot possess contradictory features. Quantum physics, in fact, may well imply the physical nature of conscious experience. Thus, the result is that at least double-aspect theory, which is the view that the mental and the physical are two aspects of, or perspectives on, the same neutral substance would be refuted. Furthermore, the mind/brain identity theory would also be refuted because experiences are not just brain processes, but they are two distinct entities, especially when the brain is a cause of experiences.

\section{Mental Experiences: A Fact, or a Delusion}

In this section, I will shortly show reasons to believe in the mentality of experience, that is to say, reasons to describe experience as mental, i.e. non-physical. In order to answer the question whether the mentality of experience is a fact or a delusion, we must first examine what experience is. Although the existence of experience seems to be clear, qualia arguments "emphasize the impossibility for materialist accounts to properly incorporate the quality of the subjective experience of a mental state, the "what it is like" (Nagel 1974) to be in that state" (Atmanspacher 2015). They argue there is something in human person that cannot be described in physical terms, which, of course, means that experience would not be entirely physical. At first, we must focus on the internality of human person. For if there is no human, then there is no human experience inside, since we have no grounds for believing in our experiences expect when we exist.

I understand internal phenomena to be things such as 1) mind and consciousness, 2) perception, thought, or experience, and 3) sensory things of attention like colors, shapes, sounds, mental pictures, hallucination and illusion, dreams, odors, tastes, or touches-now, I do not expect them to exist without any justification. If internal phenomena exist, they must be dependent on a subject who possesses them because internality implies something within which the phenomenon occurs. Even though contemporary philosophy and cognitive science consider psychological phenomena to be physical because they are related to brain activity, there are however many who believe in the existence of mental, non-physical phenomena, but what reasons make them to believe it so is an essential question. Human persons have experiences, and they reveal objects. If this human person, therefore, does not exist, there is no such thing as his or her experiences. Knowledge, by contrast, at least empirical knowledge, is derived from experience when searching for information of the world. Yet, certain general features of objects (e.g. persistence, necessity, or causal relationships) cannot be derived from the experiences we have of them, which is based on Kant's acceptance of David Hume's argument. In transcendental knowledge, Kant argued, the intellect generates the structure of objectsexperience here is the object of inquiry.

We assume as evident the existence of experience, but a claim for the existence of experiences may follow from a claim that a complete account of the physiology of human persons omits their subjective experiences and non-physical properties (qualia). A well-known example is 
Frank Jackson's knowledge argument against physicalism. Jackson's Mary is a scientist who knows everything there is to know about the science of color but has never experienced color. The question that Jackson raises is: once she experiences color, does she learn anything new? Jackson claims that she does. (Jackson 1982) These phenomena, which are first-person experiences, cannot be explained via a third-person account of brain structure or brain function. However, that experiences seem to have non-physical properties of its own is the introspectionbased claim² ${ }^{2}$ (Nagel 1974; Jackson 1982, 1986; Smith 2013, 14-17; Wendt 2015, 15-16, 110) The argument for the existence of non-physical properties of experience may be also presented as follows.

1) I directly know non-physical entities which appear in experiencing them.

2) I do not directly know material processes.

3) Knowledge is not a material process.

4) Therefore, based on Leibniz's law, to know non-physical entities which appear in experiencing them is not a material process (from 1,2,3). (This may be a masked man fallacy, even though the $3 \mathrm{rd}$ premise is true. The premises are true, but the conclusion is untrue if nonphysical properties of experience are material processes, and I do not know this fact. On the other hand, if the cause of non-physical properties of experience is material processes, then they are not identical, and the conclusion is true.)

What I found is the existence of these non-physical properties of experience in my mind by means of introspection. That is to say, because of observation of my own internal processes I have beliefs about the flow of thoughts and sensations, both of which are subjective experiences. Before we go farther it is essential to notice one thing. If the brain causes mental acts and states of consciousness, subjective experiences, and the phenomenal characters of experiences, then the brain does not exclude the human person's internal mind. My conscious introspection reveals my own private lived experiences by living through them and by interpreting them from the inside.

Speaking of experiences as our experiences guarantees the certainty that every experience is dependent on the subject who feels it. To make this statement plain, let us examine more exactly the modality and content of experience. We can individuate an experience by mentioning its modality and content. The senses like vision or hearing are modalities of perceptual experience; the content of experience is the object of awareness that is experienced, such as, for example, a visual experience (modality) of the sun (content) (Dancy and Sosa 1992, 125-127; Leon 1987). But that individuation is loose because it is a type of a psychological state of visual experience: it must be someone's vision about the sun, Jack's or Jill's, and thus it would become a particular psychological state. The existence of the content of experience requires the modality of experience, the owner of the experience, and, probably, the external environment. The conclusion leaves open what experience is in essence, still. Is experience a substance or intangible "stuff"?

It remains to ask whether there are any general, transcendental arguments enabling us to say that, if experiences are real, they must be of such and such a nature. Introspection may confirm the beliefs that flows of visual sensations, pains, and thoughts, and the like exist, and they exist

2 Introspection means the observation of one's own internal, psychological, and emotional states. 
as mental entities. Many philosophers, such as Wittgenstein, Ryle, Feyerabend, Rorty, and Churchland, who completely eliminate mental states (Wittgenstein 1953; Ryle 2000; Feyerabend 1963a, 1963b, 1969; Rorty 1965, 1970; Churchland 1981, 1984, 1985), and Place and Feigl, who assume states of the mind are identical to states of the brain (Place 1956; Feigl 1958), have objected to this statement by claiming that the "flows" of mind are nothing more than the material brain processes. Their reasons, however, vary-I will show later that in the mind/brain identity theory identity seems not to be valid between experiences and brain processes. One possible argument line is as follows. If a human body is studied, and only physiological events are discovered in it, it can be stated that there are no subjective (mental) experiences and no objects of such experiences in a living body. Nevertheless, a question arises: what this finding is based on? The truth of this finding is only demonstrated by the researchers' own psychological processes, such as belief, perceptual experience, and sensation. It then follows logically that the claim "Subjective experiences and their objects do not exist" is based on the researchers' psychological processes including such experiences, which thus contradicts their findings. It is a contradiction to state "There are no mental states such as beliefs, perceptual experience, and sensation" and to believe in that general statement-do the eliminative materialists believe in their theory? The implication "the existence of experience" can be necessarily drawn from "Empirical knowledge about the external world grounded on experience", which is valid (). A researcher's psychological processes cannot be a research subject's psychological processes. The above examples lead to real contradictions. Conscious experience seems to exist and be caused. Moreover, experience is always someone's experience. However, the essence of experience remains open: from the above cannot be inferred that experience is a non-physical or mental entity, or that it has non-physical properties. Finally, we continue to explicate the nature of experience.

To know what experiences are and to know via experiences are two distinct things. Experiences must exist in order to demonstrate truth of something by means of experiences (). As I mentioned earlier in this section, experience has content and properties. For example, the content of the yellow dot-experience is a yellow dot, and one may find it stimulating. It also has a limited duration, and it changes over time when remaining in sight. Furthermore, we do not describe the yellow dot-experience in physical terms. If asking how we feel, we do not say "I don't know but look at my brain and my facial expressions". We speak about the contents of our dreams, images, emotions, moods, visual sensations, and the like. In summary, introspection and the inadequacy of human physiological description act as evidence of the existence of experiences.

However, these two justifications-introspection and the inadequacy of complete human physiological description - do not prove the existence of the mentality of experience.

1) Thoughts, experiences, images and other mental states are demonstrated by introspection.

2) Therefore, there are mental states (from 1).

3) There are mental states.

4) Therefore, the description of human brain and other physiology remains incomplete (from 1, 2, and 3). 
It seems clear that we have conscious states, as demonstrated by introspection, or observation of one's own psychic and emotional processes. However, it does not seem obvious that introspection demonstrates that these states are, ontologically, mental, or non-physical. There are experiences, but why they would not be physical quantum phenomena is our question in the following pages.

Ever since the 1950s, many philosophers, such as Wittgenstein, Ryle, Feyerabend, Rorty, and Churchland, have argued that the events of mind are physical brain processes. In the next section, I will examine the physicality of experience from a new point of view, which is not much explored. Because, according to quantum consciousness theorists, classical Newtonian mechanics does not explain conscious experience, quantum mechanics may explain it. Human beings can be said to be psychic and physical entities, but if quantum mechanics is true throughout the universe, the human beings are also made up of quanta like all other substances and natural phenomena. Thus, experience can also be a quantum phenomenon within a quantum human and thus physical.

\section{Experience as a Physical Quantum Phenomenon: A Possibility}

In the preceding section, we found some reasons to the existence of experience, but which do not guarantee the non-physical nature of experience. Because of observation of my own internal processes, I have true beliefs about the flow of thoughts and the flow of sensations, both of which are subjective experiences. The question we must consider in this section is: does introspection fail? Subjective experiences appear non-physical, when we take the inner perspective within ourselves, yet we may be deceived by introspection. Really, subjective experiences are physical: we have only compared them to material pieces and have concluded that they are ontologically separate, incorrectly. Reason-based arguments lead us towards the physicality of experience, although we cannot experimentally confirm it. We can argue that if in the nature all phenomena are physical, and people are also natural creatures, then it follows that conscious experiences are also physical phenomena. In this section, I ask whether there is evidence for the claim that a conscious phenomenon like experience is a quantum phenomenon. To this question science does not give much answer, and when science gives, it is incomplete it is true, because, especially in quantum physics, there is not much experimental research on consciousness or experience. However, some studies have been made, which we look at initially.

A serious attempt to understand human mind and conscious phenomena, including experience, from the point of quantum physics is Alexander Wendt's inquiry Quantum Mind and Social Science from 2015. His one central claim is "All intentional phenomena are quantum mechanics"-according to him, that goes both for the private thoughts inside our heads and for public or collective intentions like norms, culture, and language, which we might generically call institutions (Wendt 2015, 149). He develops the human quantum model that would undo the classic model of human beings as material machines or zombies, which assumes nonexistence of consciousness (Wendt 2015, 3-4, 8-9, 149-153, 207-9). I focus here on how Wendt's reasons to claim that subjective experience and conscious phenomena are quantum phenomena, or quantum mechanical (Wendt 2015, 5). His starting point, I consider, is meaningful: in natural sciences, quantum physics or quantum theory has shifted away from classical Newton physics. There has been, without question, a shift from one scientific tradition to another, but the transition has not been visible in humanities and social sciences. Human beings and social life are evidently explained by scholars under the classical, Newtonian world 
view. Moreover, the causal closure of physics, which all social scientists should agree, Wendt says, implies that the social (and all other) sciences are subject to a physics constraint: no entities, relationships, or processes posited in their inquiries should be inconsistent with the laws of physics. We must notice that, according to Wendt, unlike classical physics, quantum physics does not rule out the possibility that mind is an elementary feature of reality. Wendt's personal belief is that human beings really are quantum systems. (Wendt 2015, 2-7, 9-12) It will help us in considering about Wendt's argumentation when we know what he means with 'consciousness'.

Wendt, in fact, defines consciousness as the experiential aspect of mind, the feeling: he will treat 'consciousness' and 'experience' as synonyms, which, as said above, are quantum phenomena for Wendt $(2015,15)$. The object of social sciences is a human being who is conscious-I accept this claim-, but, claims Wendt, according to positivists and interpretivists of social sciences, it would be preferable to ignore consciousness. Both positivists and interpretivists want to ignore consciousness because of its subjectivity, in other words, consciousness or subjective experience is not public and shared. (Wendt 2015, 18-19) Wendt, by contrast, argues for intentional states, including collective intentions: the mind's states are directed to a particular object; they are about something, i.e. "aboutness". ${ }^{3}$ Intentionality depends on consciousness-how a machine can be intentional, if it has no consciousness, is a meaningful enigma. (Wendt 2015, 19-20) Wendt's argument is therefore as follows: if there is an intentional state, then there is also consciousness. Most animals have intentional states and are therefore goal-oriented, but I do not believe, and Wendt does not believe, that the machine has intentional states and consciousness: my computer is hardly intentional in any way, even if the computer robot sometimes "infers" what I am going to write. (Wendt 2015, 20)

Wendt, thus, accepts subjectivity and intentionality, and consciousness (Wendt 2015, 28). The idea is that the quantum theory would explain consciousness and intentional phenomena better than classical physics: "What if the physics of the mind is not classical, but quantum - not in the trivial sense that all of reality is quantum, but in the substantive sense that consciousness itself is quantum mechanical?". (Wendt 2015, 28-30) Hence, really, a good question is: is consciousness, or experience, a quantum phenomenon? The other question, which is meaningful, is: how does quantum brain, which is said to be a quantum computer, causes quantum consciousness? (Wendt 2015, 30-31) What reasons he then provides for the view that consciousness or experience is a quantum brain-induced quantum phenomenon? This question is of the utmost importance. ${ }^{4}$ At present, everyone seems to admit that this issue cannot be resolved by empirical tests. Still, on the other hand, we do not have an empirical test to verify that there are non-physical phenomena, such as mental experiences. It is of the utmost importance for if we cannot be sure of the nature of experiences, we shall be left alone and ignorant-we seem to know that experiences and consciousness exist, but their essence is a mystery. The transcendental argument could be that the association of the universal quantum quality to the experience we are aware of makes us to discover the quantum of experience.

\footnotetext{
${ }^{3}$ I doubt the statement that all the states or experiences of mind, are intentional, that is to say, about some object or directed to some object, as has been assumed in phenomenology. A person may have experience, fear or anxiety vaguely without being subjected to any specific matter. The person cannot identify the object of his or her experience because it does not exist.
}

4 Two modern mystery of science are: 1) consciousness and 2) quantum mechanics (Wendt 2015, 39). The primary questions about quantum theory deal with metaphysics, not physics, says Wendt (2015, 39-40). On the other hand, nobody understands the quantum theory, and Wendt's goal here is to give an understanding of why we do not understand quantum mechanics (Wendt 2015, 40). 
Namely, the "Experience is a quantum mechanical" cannot be derived from the experience itself - however, below I suggest that it would be possible to observe the quantum nature of experience.

In chapters 5 and 6 of the part 2, Wendt should give reasons for quantum consciousness theory, and thus justifications for quantum cognition, experience, and subjectivity:

Quantum consciousness theory builds on these intuitions by combining two propositions: (I) the physical claim of quantum brain theory that the brain is capable of sustaining coherent quantum states (Chapter 5), and (2) the metaphysical claim of panpsychism that consciousness inheres in the very structure of matter (Chapter 6). Of these claims it is the second, panpsychism, which does the crucial work in explaining consciousness. (Wendt 2015, 92)

The classical worldview, continues Wendt, is unable to explain a phenomenon which we all know intuitively is real-first-person experience - and as such, philosophers must find ways to deny, dismiss, or deconstruct it instead. Since quantum consciousness theory purports to offer such an explanation, there is no reason to embrace that taboo, the taboo on subjectivity here. (Wendt 2015, 93) The contributions of this classical model have been huge; but it has always been assumed that the computations going on inside our heads are classical. Quantum brain theory challenges this assumption by proposing that mind is actually a quantum computer-it seems to me that this quantum brain theory implies the quantum consciousness theory in Wendt's thoughts. If quantum brain theory is true, then our model of mind would be similarly radically altered, irrespective of the issue of consciousness. (Wendt 2015, 95)

Wendt then describes how quantum brain theory hypothesizes quantum processes:

On this view, information from the environment is continuously transformed from the macro- to the micro-level, and then channeled back upward into an "internal quantum state," a decoherence-free sub-space of the brain within which quantum computational processes are performed. (Wendt 2015, 95-96)

As, and this is true, nerve cells are not smaller units to explain consciousness, then, perhaps, quantum theory is needed to explain the behavior of the nervous system, concludes Wendt (Wendt 2015, 96). Quantum brain theory proposes the firing of individual neurons of a brain is affected by quantum processes, and possibly quantum effects at the level of the whole brain (Wendt 2015, 97). A particular kind of quantum coherence, Bose-Einstein condensation, might be possible within individual cells, and from there perhaps extend to the whole brain, and be the physical basis of consciousness - the quantum consciousness theory looks at the inside of individual cells, which must be taken into account, while neuroscience assumes intercellular networks to be the mechanism from which the processes of mind arise. Wendt refers to Herbert Fröhlich's research in 1968, even though the "Fröhlich effect" has been elusive. That is to say, it remains unclear whether the effect can exist in living systems - Stuart Hameroff and Roger Penrose continued of Fröhlich 's work in the early 1990s, focusing on tiny bits of neurons called microtubules, which we will see below. ${ }^{5}$ Another tradition is the Umezawa tradition originated from an article by Luigi Ricciardi and Hiroomi Umezawa from 1967, of which central idea is that suffusing the brain's neural network is a quantum field, the dynamics of which drive its elements into coherent motion (solving the binding problem) and keeping them there (solving the stability problem) (Wendt 2015, 101-102). This chapter 5 actually deals with quantum brain

\footnotetext{
${ }^{5}$ The Neuron Doctrine tells us to look for the physical basis of consciousness in relations between neurons-neural networks-rather than inside them. However, neurons themselves are fabulously complex. (Wendt 2015, 98-99)
} 
theory and its relationship with the neuroscience of classical physics ${ }^{6}$. Let us examine next Chapter 6 if it has evidence for believing that consciousness, i.e. experience, is quantum physical.

Quantum theory makes the nature of matter as much a problem as mind. If quantum matter is "matter" at all, in short, then it is a thoroughly de-materialized matter, far from what we ordinarily imagine matter to be. (Wendt 2015, 112) In fact, Wendt argues for panpsychism, and the next reference suggests that mind would then be a physical entity: "mind is neither reducible to matter nor emergent from it, but in matter all the way down - which in turn obviates the need to posit dualism's two substances in response to materialism's failure to explain consciousness (Wendt 2015, 112). He thinks that subjectivity is intrinsic to matter, or more precisely "proto"subjectivity is the antecedent to subjectivity before the latter is organized into living matter (Wendt 2015, 115). Attributing subjectivity to non-human organisms, and to other human organisms than me, is difficult, but he suggests through a combination of empirical evidence and logical reasoning that we can arrive at a rational answer (Wendt 2015, 117). He thus continues to argue that, in some sense, all organisms, including bacteria, cells, and plants, are subjects and therefore conscious (Wendt 2015, 116-119). For showing that mind and matter are continuous, the answer, according to Wendt, is to exploit the causal gaps in quantum theory by identifying subjectivity (or more precisely, proto-subjectivity, since we are not talking here about living matter) with the formalism of quantum mechanics, only viewed now from the inside, as intrinsic to matter, rather than from the outside (Wendt 2015, 119). Wendt describes a lot how cognition, experience, and will are quantum subjects, but he does not provide many reasons for accepting that they really are (unless quantum physics and quantum theory are considered as reasons) (Wendt 2015, 119-123). He argues later in chapter 6 that quantum coherence $^{7}$ in the brain explains the unity of consciousness at the human level (Wendt 2015, 131). I consider that he argues here through theories, but if a theory contains concepts that cannot be derived from experience, then the theory cannot be tested in principle. However, as Kant thought, these concepts can be meaningful to combine with experience and its object, such as concepts of unity, quality, causation, and persistence. It may make possible for us to detect experience as consequence or quantum-physical.

In my view, Wendt's arguments are based solely on second-hand sources, quantum physics, and quantum brain theory, which is the weakness of his work. He could provide a concrete example of quantum mechanics that would increase the understanding of the "causal mechanism" in the quantum system. But, according to quantum consciousness theorists, quantum physics explains better conscious states than classical Newtonian physics, neuroscience, or social sciences explain. Some scientists, by contrast, criticize the hypothesis where consciousness or experience is a quantum phenomenon, leading to a conclusion that internal psychological states could affect world events. For example, internal thinking could affect the natural phenomena of the physical reality-I have no such assumption in this article. For example, physicist Lawrence Krauss says in an interview:

\footnotetext{
${ }^{6}$ That the brain consists of quanta like the whole physical reality consists, but quantum phenomena have nothing to do with the brain's activity, is a strange statement from neuroscientists and philosophers. Quantum brain theory, to my mind, is at least a possible and legitimate research perspective. See Wendt 2015, 102-108.

${ }^{7}$ Quantum coherence refers to a situation in which the wave functions of two or more particles are entangled, such that they form a superposition that can be described with one equation. Concretely, this means the properties of the system's elements are correlated non-locally, so that a measurement on one instantly tells us something about the others. (Wendt 2015, 98)
} 
We are connected to the world by many things: by light and sound and heat. We do, at subatomic scale, behave quantum mechanically. But we behave like classical objects for a reason: We're big, we have lots of particles, they interact. All the weirdness of quantum mechanics gets washed out on the scale that we can experience. That's why we experience a classical world. (Boyle 2015)

I ask what that experience is. Is experience something that any physical theory cannot explain, that is to say, it would be something non-physical, even if it would merge with the brain's neural cells? It is, however, a matter of human internality, of the body, which implies that inner thinking does not directly affect the environment, but through the body we can influence the environment; I do not pour the tree to the ground by thinking about it but for instance by sawing the tree. Let us next see how other quantum consciousness theorists characterize consciousness and experience.

An American scientist and philosopher David Bohm developed a theory of the relationship between mind and matter, which would participate with each other, and they do not interact: he said that this theory does not divide mind from matter, as Descartes' view did, and where mind and matter interact somehow through action of God, but connects them non-dualistically (Bohm 1990, 271, 275-6, 283-5). Bohm's theory implies that consciousness, or mind, is a quantum phenomenon, yet, surprisingly, mental. In the beginning of his work, he assures that "the quantum theory denies the mechanistic (Newtonian) conceptual framework which has thus far implicitly justified the notion that mind is of such a nature that it can have absolutely nothing to do with the laws of matter" and concludes, in the end of his work, that "The content of our own consciousness is then some part of this over-all process. It is thus implied that in some sense a rudimentary mind-like quality is present even at the level of particle physics, and that as we go to subtler levels, this mind-like quality becomes stronger and more developed" (Bohm 1990, 272, 283). These statements, in my opinion, would refer to mind that is subject to the laws of physics and also physical, if it is present when the particles are present. According to Bohm, mind and matter are closely analogous: "Or to put it differently, the general implicate process of ordering is common both to mind and to matter" (Bohm 1990, 273). His causal interpretation of quantum theory provides a basis for a non-dualistic theory of the relationship of mind and matter. The first step, said Bohm, is to assume that the electron is a particle, following a trajectory, as a planet around the sun, but always accompanied by a new kind of quantum field - fields are familiar to us like magnetic field. (Bohm 1990, 275-6) The quantum field, however, is different than fields in Newtonian physics:

In physics, a potential describes a field in terms of a possibility or potentiality that is present at each point of space for giving rise to action on a particle which is at that point. (Bohm 1990, 276)

Bohm, furthermore, argued that the quantum field can also be represented in term of a potential, which he called the quantum potential:

But unlike what happens with electric and magnetic potentials, the quantum potential depends only on the form, and not in the intensity of the quantum field. Therefore, even a very weak quantum field can strongly affect the particle. It is as if we had a water wave that could cause a cork to bob up with full energy, even far from the source of the wave. Such a notion is clearly fundamentally different from the older Newtonian ideas. For it implies that even distant features of the environment can strongly affect the particle. (Bohm 1990, 276) 
After explained this by an example, by the two slit interference experiment ${ }^{8}$, Bohm presented a new notion of active information:

The word in-form is here taken in its literal meaning, i.e. to put form into (rather than in its technical meaning in information theory as negentropy). One may think of the electron as moving under its own energy. The quantum potential then acts to put form into its motion, and this form is related to the form of the wave from which the quantum potential is derived. (Bohm 1990, 279)

Bohm's example was a ship on automatic pilot guided by radar waves. These waves do not mechanically push and pull the ship. The form of the waves is picked up, and with the aid of the whole system, this gives a corresponding shape and form to the movement of the ship under its own power. (Bohm 1990, 279) The task is then to extend this notion of active information to matter at the quantum level, and it has implications for mind-this quantum field and quantum potential are important later when consciousness is considered as a field, as a nonmaterial field; for example, a single experience would give a "kick" to the body parts. The notion of active information implies the possibility of a certain kind of wholeness of the electron with distant features of its environment (Bohm 1990, 280).

Bohm was going further by asserting that the whole notion of active information suggests a rudimentary mind-like behavior of matter, for an essential quality of mind is just the activity of form, rather than of substance:

Thus, for example, when we read a printed page, we do not assimilate the substance of the paper, but only the forms of the letters, and it is these forms which give rise to an information content in the reader which is manifested actively in his or her subsequent activities. A similar mind-like quality of matter reveals itself strongly at the quantum level, in the sense that the form of the wave function manifests itself in the movements of the particles. (Bohm 1990, 281)

When Bohm applied an autopilot ship, radio waves, and quantum processes in elementary particles to the aspect of thought and subjective experience, then suspicion wakes up against this analogy. How does the non-material field of mind, or active information, affect a thought and thereby activate body functions? The question refers to a transcendental statement that is not due to experience. (Bohm 1990, 281-2)

Bohm assured as follows:

It seems clear from all this that at least in the context of the processes of thought, there is a kind of active information that is simultaneously physical and mental in nature. Active information can thus serve as a kind of link or 'bridge' between these two sides of reality as a whole. (Bohm 1990, 282)

These two sides, according to Bohm, are inseparable. Information contained in thought, which we experience to be on the mental side, is also a related to neurophysiological, chemical, and physical activity (Bohm 1990, 282). Next Bohm's remark is special. Our thoughts contain a large amount of information content that, as Bohm said, we can look at the higher level of mental activity, as if we looked at a material object. From this level, a subtle level of information may arise, the significance of which is an activity that organizes the original set of information

\footnotetext{
${ }^{8}$ One of the most famous experiments in physics is the double slit experiment. It demonstrates, with unparalleled strangeness, that little particles of matter have something of a wave about them and suggests that the very act of observing a particle has a dramatic effect on its behavior. See about this: Marianne 2017.
} 
into a larger entity - 'subtle' or its Latin root, 'sub-texere', which means 'finely woven'. However, this kind of subtle information can be viewed at a subtler level of mental activity, and in principle, this can continue indefinitely. Each of these levels can be seen on the material side where Bohm proceeded:

From the mental side, it is a potentially active information content. But from the material side, it is an actual activity that operates to organize the less subtle levels, and the latter serve as the 'material' on which such operation takes place. Thus, at each level, information is the link or bridge between the two sides. (Bohm 1990, 282)

In the last section of his article, Bohm considered how the above is related to the quantum theory. He claimed that because the quantum potential seems to be information whose activity is to guide the "dance" of the electrons, there is a basic similarity between the quantum behavior of a system of electrons and the behavior of mind - an information field guides the behavior of mind. (Bohm 1990, 283)

Quantum processes can also be capable of extension to great levels of subtlety (Bohm 1990, 283). What Bohm now concluded is that there are not two processes, but both are one and the same. Thus, there is no real division between mind and matter, psyche and soma. That is how we ended up with how we began to handle Bohm's theory: mind and matter would participate with each other, they do not interact; Bohm said that this theory does not divide mind from matter, as Descartes' view did, and where mind and matter interact somehow through action of God, but connects them non-dualistically. Bohm's idea, according to a philosopher of mind Paavo Pylkkänen $(2015,333)$, is that mental processes are carried by subtle physical processes, fields that are analogous to, but more complex than the quantum field. It is clear that Bohm does not contribute to identity between the experience of the mind and the possible neurophysiology, which would also require the concept of necessity. It is impossible to see the necessity in experience, and thus it must be a transcendental act.

A statement of quantum consciousness theorists is that neuroscience and classical Newtonian physics have not solved the problem of consciousness. Neuroscientists, presumably, consider it clear that the brain can be explained by classical Newtonian terms. This Bohm broke: quantum theory can help us understand what is happening in a neural process. If consciousness or experience is still thought of as a mental phenomenon, it is confusing how quantum theory could increase understanding of consciousness or experience because quantum theory is to explain physical phenomena. This is how Hiley and Pylkkänen (2005, 20; Pylkkänen 2015, 333-335) assume, even though the non-physical phenomenon is not explained by any theory of physics. As Hiley and Pylkkänen (2005; Pylkkänen 2015, 333-335) argue, all neural processes are not explained by classical Newtonian theory, yet, to my mind, no theory of physics explains the behavior of a non-physical phenomenon. Mind may be thought differently, as a non-material field, in Bohm's, Hiley's, and Pylkkänen's idea of the relation between mind and matter (Hiley and Pylkkänen 2005, 20-21). Theoretical physicist Roger Penrose and anesthesiologist Stuart Hameroff also aimed to explicate neural processes of the brain by quantum mechanics, and thus also to explicate experience and consciousness. In his The Emperor's New Mind (1989), Penrose challenged the conventional wisdom that human brains are computers; that human brains are computational as David Marr (1982) argued. Penrose went further in this work and proposed that human brains were not even quantum computers, but that a deeper understanding of physical laws would be needed to explain consciousness. Penrose believes that consciousness is not computational. Our awareness is not simply a mechanistic byproduct, like something we can make a machine do. In particular, Penrose thinks the answer to consciousness may lie in a 
deeper knowledge of quantum mechanics-here, I do not examine the view that mind is not computational.

Penrose (1994, 1995, 1996a, 1996b) and Hameroff (1995, 1996a, 1996b, 1998a, 1998b) proposed in the mid 1990's that consciousness depends on biologically "orchestrated" coherent quantum processes in collections of microtubules within brain neurons, and, in an article from 2014, they reviewed this the "Orch OR" theory in light of criticisms and developments in quantum biology, neuroscience, physics, and cosmology (Hameroff and Penrose 2014). Alexander Wendt, for example, describes Penrose's and Hameroff's view as follows. The Neuron Doctrine, says Wendt, tells us to look for the physical basis of consciousness in relations between neurons - neural networks - rather than inside them. However, neurons themselves are fabulously complex. The proteins are organized in web-like structures making up the "cytoskeleton," which gives neurons their form and regulates their connections. Microtubulesso named because they look like hollow tubes - are the building blocks of the cytoskeleton, and there are typically thousands in a single neuron. However, according to Wendt, there is still the question of whether any computation going on within microtubules is quantum or classical. Here the case for a quantum answer begins by pointing out that microtubules are at precisely the right scale to mediate between quantum processes at the sub-atomic level and classical neural computation. From there the argument highlights several intriguing features of microtubules that suggest they can amplify those quantum processes into coherent superpositions at the microtubular level. (Wendt 2015, 99-100) Hameroff and Penrose, moreover, claim in their article that the "Orch OR" theory-Orchestrated objective reduction (Orch-OR) is a hypothesis that consciousness in the brain originates from processes inside neurons - proposes quantum computations in brain microtubules account for consciousness, and, moreover, microtubule "quantum channels" in which anesthetics erase consciousness are identified. Again, I will ask here whether consciousness or experience is a quantum phenomenon and by what reasons we can believe so.

By contrast to Science/Materialism and Dualism/Spirituality, Hameroff and Penrose believe that consciousness results from discrete physical events; such events have always existed in the universe as non-cognitive, proto-conscious events, these acting as part of precise physical laws not yet fully understood (Hameroff and Penrose 2014, 39-40). How does then the brain produce consciousness, when we assume the brain is the cause of consciousness? To this question neuroscience gives an answer, somewhat incomplete and in part hypothetical. Many scientists and philosophers view consciousness as an emergent property of complex computation among "integrate-and-fire" brain neurons which interconnect and switch at chemically mediated synapses. However, Hameroff and Penrose argue, the mechanism by which such neuronal computation may produce conscious experience remains unknown because there are many unexplained features of consciousness left. (Hameroff and Penrose 2014, 40-41) Furthermore, argue they, there is no sense of how consciousness arises from neurocomputation (Hameroff and Penrose 2014, 41-42). According to Hameroff and Penrose, finer scale intra-cellular processing, e.g. derived from cytoskeletal structures, are the means by which single-cell organisms perform cognitive functions without synaptic inputs. They claim that neurons have a rich and uniquely organized cytoskeleton, the major components being microtubules, wellpositioned and uniquely arrayed (e.g. in dendrites and soma) to mediate consciousness and regulate firing. (Hameroff and Penrose 2014, 43)

Microtubules processes may directly result in conscious awareness (Hameroff and Penrose 2014, 45), but Hameroff and Penrose do not provide evidence verifiable by observation but rather by theory for that statement. On the contrary, they mostly tell that microtubules information processing depends on interactive dipole states of individual tubulin proteins 
(Hameroff and Penrose 2014, 46). Because approaches based on microtubules information processing with classical physics faced a reductionist dead-end in dealing with consciousness, Hameroff and Penrose started considering quantum mechanics. A critical realization was Penrose's idea that all mental processes are not computational, like certain aspects of consciousness, such as understanding, but 'non-computable', appealing to Gödel's theorem and other considerations. (Hameroff and Penrose 2014, 48-49) The non-computable ingredient required for human consciousness and understanding, Penrose suggests, would have to lie in an area where our current physical theories are fundamentally incomplete. The only serious possibility is the incompleteness of quantum theory. This incompleteness is the unresolved issue, and one way to resolve it would be to provide an extension of the standard framework of quantum mechanics by introducing an objective form of quantum state reduction - termed "OR" (objective reduction). (Hameroff and Penrose 2014, 49) The proposed connection between consciousness and quantum measurement is almost opposite, in the Orch OR scheme, but the so-called $\mathrm{DP}^{9}$ proposal suggests each OR event, which is a purely physical process, is itself a primitive kind of "observation", a moment of "proto-conscious experience" (Hameroff and Penrose 2014, 49). In section 4, Hameroff and Penrose say they are dealing with quantum mechanics and awareness. However, according to my observation, consciousness is not mentioned much. (Hameroff and Penrose 2014, 48-58) They argue, generally speaking, more in a way that "For the Orch OR and consciousness to occur, such and such have to do this and this" (Hameroff and Penrose 2014, 54). It is true that there is clear evidence for coherent microtubule quantum states at brain temperature, like research has shown other warm quantum effects, for example, in bird navigation and sense of smell. But the actual mechanisms underlying the production of consciousness in a human brain is hard to be deduced from these previous research of quantum effects and from Hameroff's and Penrose's theoretical considerations. (Hameroff and Penrose 2014, 55-58, 59-66, 68-73) The Orch OR provides a possible way to account for frequent moments of conscious awareness and choices governing conscious behavior (Hameroff and Penrose 2014, 59), and possible evidence for a belief that a quantum effect is necessary for consciousness to exist. Their theorems are not experimentally detectable, but so are the counterarguments and philosophical theories undetectable too. Nor do Penrose and Hameroff identify the identity between consciousness and neuronal function, and furthermore, identity and necessity cannot be derived from experience. The use of the concept of causation would be related to a transcendental statement: the intellect associates them with experience and form a statement, such as "Brain processes produce conscious experience". In the future, by contrast, if quantum technology advances to the point that direct tests of quantum theory on macroscopic objects are possible, our discussion does give a clear motivation for carrying them out on animals and humans-I will argue about this issue soon below. (Kent 2018, 8)

I conclude that even if experience or consciousness were not quantum phenomena, if not Bohm (Bohm 1990, 283-5; Pylkkänen 2015, 9), nevertheless, many others, such as Hameroff and Penrose, think that they are physical, not something non-physical — and many philosophers still talk about mental processes and states of consciousness. If they are quantum phenomena, which is a plausible conjecture, their behavior is subject to the laws of physics and requires, perhaps, a different physics theory than quantum mechanics - how a physical event affects a nonphysical phenomenon, is obscure, and thus quantum consciousness conjecture seems to be meaningful.

\footnotetext{
${ }^{9}$ The Diósi-Penrose proposal gives an objective physical threshold, providing a plausible lifetime for quantum superposed states (Hameroff and Penrose 2014, 49).
} 
The mathematical models of quantum physics, which also are meaningful, cannot directly apply to consciousness and experience, whether they are introductory to university students or more profound theoretical physics models. From the explanations of Bohm's quantum physics, for example, cannot directly deduce what consciousness or experience is in essence; the same is valid with Hameroff's and Penrose's line of thought.

Hypotheses have been proposed about ways for quantum effects to be involved in the process of consciousness, but even those who advocate them admit that the hypotheses remain empirically unproven, and possibly unprovable. Some of the proponents propose experiments that could demonstrate quantum consciousness, but the experiments have not yet been possible to perform. However, to my mind, it is a different matter to explain consciousness or subjective experience by quantum mechanics and laws of quantum mechanics than saying that consciousness or subjective experience is essentially a quantum phenomenon, and thus a physical entity like the whole human body is, or water is $\mathrm{H} 2 \mathrm{O}$ in the physical world. Physicists consider hearing claims of quantum consciousness or quantum experience as nonsense (Ouellette 2016), but why is there no reason to conclude that conscious experience is a physically possible entity, probably a quantum phenomenon? If any new physics does not explain the essence of consciousness or subjective experience, then it cannot be deduced from that that consciousness or subjective experience is not a quantum phenomenon. Still, if we had quantum technology to detect quantum events, then we could observe whether the subjective experiences are physical quantum phenomena. The reason-based argument could thus be as follows.

We see macroscopic bodies with eyes; we see the cell level through the microscope; we might see, let us suppose, the human mind and its experiences and the human body how they are the same ontological kind of entities, physical quanta, through a quantum technological instrument revealing quantum-level phenomena. That is to say, we might see the quantum human as a whole. Therefore, conscious experience would be a quantum phenomenon. Human is not composed of matter, i.e. of body, and of non-physical mentality, i.e. of mind, assuming that the assumption is true.

In this section, the weakness is that it cannot be shown by empirical tests or experiments that experience is a quantum phenomenon. On the other hand, reason-based arguments bend us in this direction rather than the view that experience would be entirely different from other physical phenomena by nature. Introspection really reveals experiences and other psychic states, but it cannot be deduced from that fact that they are not physical quantum phenomena but are identical to states and processes of the brain. In the following section, I will show that, at least, conscious experience is not both a physical and non-physical feature.

\section{If Experience Is a Quantum Phenomenon, It Refutes Double- aspect Theory}

The last two sections appear to be in contradiction. Firstly, experiences were argued to be mental or non-physical; they seem to have non-physical properties, tells the knowledge argument. But secondly, there seems to be reasons for the view that conscious experience is a quantum phenomenon, and thus implying physicality. In this section, I will argue that experience cannot be both a physical and a non-physical or mental phenomenon because no entity can possess the opposite properties. Because experience as such does not contain contradiction, such an argument seems to be a transcendental argument. Thus, for example, 
double-aspect theory ${ }^{10}$ is refuted, which means that the neutral substance would be both nonphysical and, on the other hand, also physical, such as the two sides of the coin. That theory, however, requires the mental and the physical to be inseparable and mutually irreducible (though distinct). It is noteworthy that, however, experience is not a substance like a coin but some animate creature's feature or an event within the creature. I will also show later that the $\mathrm{mind} /$ body identity theory is implausible when experiences are brain-induced: the brain and its processes and experiences are two distinct entities, not one. In the mind/body identity theory, their analogues are false, and identity seems not to be perceptible but transcendental between experiences and brain processes.

To make the difficulties of double-aspect theory plain, let us concentrate attention on two sets of beings. It is plain that two contrary properties cannot both be true: it cannot be true that "all $\mathrm{S}$ are P" and "all S are non-P." The truth of one of these contrary propositions excludes the truth of the other. For example, no entity is both physical and non-physical. Some entity might be physical and mental, but one cannot call it wholly non-physical because it also is physical. Physicality and mentality, however, are not evidently contrary properties, as non-physicality includes more than mentality. Abstractness and conceptuality are also non-physical properties, such as transcendental concepts of unity, necessity, causality, and quality. Mentality and nonmentality are contrary properties. These abstract distinctions also apply to concrete cases, of course. Something is both circular and yellow, but one cannot then call it non-circular because it is circular, and it would be a contradiction. Circularity and yellowness are not contrary properties but, for example, being colored and being colorless are. When looking more deeply, is it indeed impossible that a substance is both A and non-A (the contrary)? For example, let us say that one set includes non-physical entities, and other set physical entities. Could we, then, give an example in which one thing yellow dot-experience is both non-physical and physical? There is an entity present in both sets, and thus these sets would intersect. Therefore, the question "What is a yellow dot-experience?" would produce the answer "The yellow dotexperience is a physical entity", which is an identity statement. However, if we form the objection "The yellow dot-experience is a non-physical entity", it would be untrue and contradictory because "A physical entity is a non-physical entity" is untrue and contradictory. Nevertheless, for some philosophers, the statement "The yellow dot-experience is a physical entity" does not seem to be a true identity statement because the yellow dot-experience cannot be wholly described in a materialistic way, as stated in the second section. The statement "The yellow dot-experience is a non-physical entity" would be true because experiences have nonphysical properties - although it is not clear whether Nagel and Jackson would consider experience entirely non-physical. (Nagel 1974; Jackson 1986; Smith 2013, 14-17) Therefore, sets of non-physical entities and sets of physical entities do not intersect. Thus, it seems that experience and the content of experience are not both non-physical and physical because contrary properties cannot coincide in one and the same subject simultaneously. But let us next consider double-aspect theory: a thing, experience is both mental and physical, or experiences, which are neutral "stuff", have two aspects, the mental and the physical. If, generally, experience is a physical quantum phenomenon, as partly argued in the third section, then experience is not a mental or non-physical phenomenon because experience cannot have contrary features.

\footnotetext{
${ }^{10}$ Double-aspect theory have been advocated by philosophers such as Baruch Spinoza and Arthur Schopenhauer. Spinoza believed that the Existence had two aspects, Extension and Mind, which together were to be taken as two of an infinite set of attributes comprising God (or, Nature). Schopenhauer considered the fundamental aspects of reality to be Will and Representation.
} 
The claim is that experience cannot be described in a materialistic way, i.e. it would have nonphysical properties. Because mentality is often understood as non-physicality, a mentalphysical thing, when experience is interpreted in the context of double-aspect theory, implies non-physicality. Furthermore, it also implies physicality, and thus experience can be described in a materialistic way. Yet, if experience is a quantum phenomenon, this would mean that, by contrast, it is physical because it is not both non-physical and physical. According to doubleaspect theory, when we look at experience under this theory, which is transcendental, conscious experience would be both mental and physical. Double-aspect theory argues that experience is an example of a neutral level that is both non-physical and physical because the level is a mental-physical thing. However, the mental-physical thing cannot be non-mental and nonphysical because this is inconsistent with the physical quantum phenomena. Experience and the content of experience have physical and subject dependent features. Moreover, the dual aspect cannot be derived from experience we are aware of; such a general feature is not perceptible. This conclusion mean that the experiences cannot have physical and non-physical properties. If Linda has only quantum properties, you can deny that she has non-physical properties because of physical quantum properties. So, we ended up with the following argument.

1) No object can possess contradictory features (self-evident).

2) Physicality and non-physicality are contradictory features (logical truth).

3) Experience is not a physical and non-physical phenomenon (from 1 and 2).

4) Experience is a physical quantum phenomenon (from the third section: a real possibility).

5) Therefore, experience is not a non-physical phenomenon (from 3 and 4).

If, generally, experience is a quantum phenomenon, as partly argued in the third section, then experience is not a mental or non-physical phenomenon. Therefore, double-aspect theory is refuted, which means that experience would not be both non-physical and, on the other hand, also physical, such as the two sides of the coin.

\section{If Experience Is a Quantum Phenomenon, It Also Refutes the Mind/Brain Identity Theory}

Above, we have noticed that quantum consciousness theorists assume that the brain is a causal cause of experiences. This assumption is also in line with the current brain research according to which the brain causes states of consciousness. By causality, which is a transitive relation, I understand there are at least two entities, not one, when experiences are brain-induced. In this section, I will argue that the mind/brain identity theory cannot be true. It also seems from the sources that the identity theorists' analogues are implausible, which I will begin with.

Some philosophers hold that though experiences are brain processes they nevertheless have fundamentally non-physical, psychical, properties, sometimes called 'qualia'. According to J. J. C. Smart, we should take the mind/brain identity theory as denying the existence of irreducible non-physical properties. The identity theory of mind holds that states and processes of the mind are identical to states and processes of the brain. Consider an experience of pain, or of seeing something, or of having a mental image. The identity theory of mind is to the effect that these experiences just are brain processes, not merely correlated with brain processes. 
(Smart 2007) Identity theorists use identities as examples in which experiences and brain processes are analogies.

Following Gottlob Frege, two terms may have different meanings but the same referent, which implies identity. "The Morning Star" and "the Evening Star" have the same referent (the planet Venus). Identity theorists use this model when claiming experiences and brain processes identical. (Smart 2007, 2-3) For example, U. T. Place (1954, 255) remarked:

The logical objections which might be raised to the statement 'consciousness is a process in the brain' are no greater than the logical objections which might be raised to the statement 'lightning is a motion of electric charges'.

An analogy between natural phenomena and the phenomena of mind fail. Experiences may be the results of the processes rather than the processes themselves. The early stages of observation or pain do not even include experience and do not come to the person's consciousness. Physical energy such as light, needle stitching or drinking coffee activates a sensory organ that puts the nerve cell moving toward the hemisphere, generating somehow a conscious experience of yellow, pain, or coffee flavor. Because the pain experience has some causes that cause it, the pain experience is not identical to these different causes (such as a needle sting). Philosophers do not seem to understand the difference between identity and causality, which I will explain next.

Causality and identity are opposite ideas. If $\mathrm{A}$ is identical to B, then $\mathrm{A}$ does not cause $\mathrm{B}$, and vice versa. Moreover, if, in a transitive relation, $A$ causes $B, B$ causes $C$, and $C$ causes $D$, then $\mathrm{C}$ is not identical to $\mathrm{A}$, which is self-evident. Identity between several things in the causal chain, in fact, is a contradiction because, as Nicholas of Cusa judiciously said, there cannot be several things that are exactly the same, for in that case there would not be several things, but the same thing itself (Nicholas of Cusa, De Venatione Sapientiae, Chapter 23). If the external entity is a cause and what appears to me directly in awareness is the consequence, then they are not identical. For example, a needle hits the skin, causing the movement of nerve cells. They pass through the neural pathways along the cortex and probably cause injection sensation, pain sensation, in my awareness. The introspection-based statement that the pain is identical to the property of the external substance is untrue. This example reveals as problematic a general claim according to which the features of experience are the features of an external substance that causes this experience. Because experience seems to occur without the person doing something, it would be a mistake to combine identity with experience and an external substance. It is not possible for two identical events or states to be in the cause-effect relation. Such a possibility would mean that event $F$ is identical to an event $G$, and the event $F$ causes the event $\mathrm{G}$ (that is not the event F), which is absurd. The existence of two identical events in the cause and the effect relation is absurd because causality is the transitive relation between an event (the cause) and a second event (the effect). I do not believe that the two labels "being sweet" and "being a sugar's property A" refers to the same entity. Sugar's ingredients affect the perceiver's sense in order to cause an experience of sweet flavor after the stimulation of the perceiver's sense. Furthermore, by doing surgery on the perceiver's senses, the perceiver's experiences may change without changing the brain process: an experience of flavor changed. And yet again, I repeat. If a set of factors (the external entity and the network of neural cells) is a cause of the immediate object of experience, or how things appear to the perceivers, then the set of factors is not identical to the immediate object of experience. In conclusion, the causality and the identity are mutually exclusive, excluding the possibility that a property of the brain is identical to the intentional object, which I am immediately aware of in perception, such as a sweet taste. The examples above inevitably lead to the following argument. 
Experience is not identical to the brain process because they are two distinct entities.

1) If $A$ is a causal cause of $B$, then $B$ is not identical to $A$.

2) If $A$ and $B$ have different properties, then $A$ and $B$ are not identical.

3) The brain process $\mathrm{A}$ is one of the causal causes of experience $\mathrm{B}$.

4) Experience $B$ is also caused by physical energy $C$ and sensory activation $D$.

5) Therefore, experience $B$ is not identical to the brain process $A$ (from 1, 2, 3, and 4).

Thus, since there are two entities, namely experience B and brain process A, there can be no one and the same entity. Experiences arise as a target for consciousness that implies nonidentity with the brain process. The mind/brain identity is also refuted. Causal theories of consciousness also make quantum consciousness theories into meaningful alternatives to explore more closely.

\section{Conclusion}

The question of what consciousness, conscious experience, or mind are in essence is currently still open in the sciences. At least a few things can be said to be merits of quantum consciousness theories. One point is that neuroscience has not been able to solve the problems of consciousness. And the second point is that both neuroscience and humanities are still looking at people from the classical Newtonian worldview; the essence of man would be unique in relation to other natural. It is a favoritism on behalf of human against other natural things. The weakness, on the other hand, is impossibility to test quantum consciousness theories. And if the theory contains transcendental concepts, such as the concept of necessity, then it cannot be even empirically tested. Scientific argumentation, especially in theoretical research, can well be based on reasoning and logic that others can then evaluate; logic can at least show how a thing is not probably in reality, such as contradictions or inaccuracies in causal observations. The merit of my result is based on a logical reasoning: experience is not consistent with doubleaspect theory. More probably it is that it is not consistent than that it would be consistent. Furthermore, the causal relationship between the brain and experiences implies the impossibility of the identity of experiences and brain processes, because causality and identity are different things. When the network of nerve cells is the cause and an experience is the effect, then there are 2 distinct entities, not 1 network of nerve cells. Therefore, the mind/brain identity theory is implausible, when experience is a quantum phenomenon caused by the brain.

The result of this article is that the mentality of experience, that is to say, the non-physicality, may been refuted. The human organism has the experience of something like, for example, itself, and, probably, this is a quantum property of human. When the two previous sections are combined, we have perceived them incompatible. If experience is a quantum phenomenon, then it is not a simultaneous non-physical phenomenon, a phenomenon associated with so-called "mind". I have argued that experience is impossible to be both a physical and non-physical phenomenon because an object cannot possess contradictory features.

Logical reasoning and science, like stated above, strongly indicate that experience is more likely to be a physical entity created by the brain than a mental entity, which implies non-identity between experiences and brain processes in the network of nerve cells. The causal conclusion from experience to brain processes is the transcendental conclusion in which causality is 
combined with experience. Because experience seems to occur without the person doing something, it would be a mistake to combine identity with experience. On the other hand, the issue does not rule out internality and externality in the line of mind and the external world. Where is the boundary of inner experience and the external world? These are the relevant questions, but they are the subject of other studies.

\section{References}

Atmanspacher, H. (2015). Quantum Approaches to Consciousness. The Stanford Encyclopedia of Philosophy. https://plato.stanford.edu/archives/sum2015/entries/qt-consciousness/. Accessed 14 April 2019.

Bohm, D. (1990). A new theory of the relationship of mind and matter. Philosophical Psychology, 3(2), 271-286.

Boyle, A. (2015). How to Spot Quantum Quackery. NBC News, November 3. https://www.nbcnews.com/sciencemain/how-spot-quantum-quackery-6C10403763. Accessed 3 December 2018.

Churchland, P. M. (1981). Eliminative Materialism and the Propositional Attitudes. The Journal of Philosophy, 78(2), 67-90.

Churchland, P. M. (1984). Matter and Consciousness. Cambridge, Massachusetts: The MIT Press.

Churchland, P. M. (1985). Reduction, Qualia, and the Direct Introspection of Brain States. The Journal of Philosophy, 82(1), 8-28.

Dancy, J. and Sosa, E. (1992). A Companion to Epistemology. Cambridge, Massachusetts: Basil Blackwell.

Feigl, H. (1958). The "Mental" and the "Physical". In H. Feigl, M. Scriven and G. Maxwell (Eds.), Concepts, Theories and the Mind-Body Problem (Minnesota Studies in the Philosophy of Science, Volume 2). Minneapolis: University of Minnesota Press.

Feyerabend, P. K. (1963a). Comment: Mental Events and the Brain. The Journal of Philosophy, 60(11), $295-296$.

Feyerabend, P. K. (1963b). Materialism and the Mind-Body Problem. The Review of Metaphysics, XVII(1), 4966.

Feyerabend, P. K. (1969). Science Without Experience. The Journal of Philosophy, 66(22), 791-794.

Hameroff, S. (1998a). Quantum Computation in Brain Microtubules? The Penrose-Hameroff 'Orch OR' Model of Consciousness. Philos Trans $R$ Soc Lond Ser A, Math Phys Sci, 356, 1869-96.

Hameroff, S. (1998b). 'Funda-mentality': Is the Conscious Mind Subtly Linked to a Basic Level of the Universe?. Trends Cogn Sci, 2, 119-27.

Hameroff, S. and Penrose, R. (1996a). Orchestrated Reduction of Quantum Coherence in Brain Microtubules: A Model for Consciousness. In S. Hameroff, A. W. Kazniak and A. C. Scott (Eds.), Toward a Science of Consciousness; the first Tucson discussions and debates (pp. 507-40). Cambridge (MA): MIT Press.

Hameroff, S. and Penrose, R. (1996b). Conscious Events as Orchestrated Space-time Selections. Journal of Conscious Studies, 3(1), 36-53.

Hameroff, S. and Penrose, R. (2014). Consciousness in the universe: A review of the 'Orch OR' theory. Physics of Life Review, 11, 39-78.

Hiley, B. J. and Pylkkänen, P. (2005). Can Mind Affect Matter Via Active Information?. Mind \& Matter, 3(2), 7 27.

Jackson, F. (1982). Epiphenomenal Qualia. The Philosophical Quarterly, 32(127), 127-136.

Jackson, F. (1986). What Mary Didn’t Know. The Journal of Philosophy, 83(5), 291-295.

Kant, I. (2010/1781/1787). Kritik der reinen Vernunft. J. Timmermann (Ed.). Hamburg: Meiner. Kant, I. (1998). Critique of Pure Reason. P. Guyer \& A. Wood (Trans.). Cambridge, UK: Cambridge University Press.

Kent, A. (2018). Quanta and Qualia. Foundations of Physics, https://doi.org/10.1007/s10701-018-0193-9

Leon, M. (1987). Character, content, and the ontology of experience. Australasian Journal of Philosophy, 65(4), 377-399. 
Marr, D. (1982). Vision. San Francisco: W. H. Freeman.

Nagel, T. (1974). What Is It Like to Be a Bat?. The Philosophical Review, 83(4), 435-450.

Nicholas of Cusa. Metaphysical Speculations. In J. Hopkins (Trans.), Six Latin Texts Translated into English. Minneapolis, Minnesota: The Arthur J. Banning Press.

Ouellette, J. (2016). A New Spin on the Quantum Brain. Quanta Magazine, November 2. https://www.quantamagazine.org/a-new-spin-on-the-quantum-brain-20161102/. Accessed 18 December 2018.

Penrose, R. (1989). The Emperor's New Mind. Oxford: Oxford University Press.

Penrose, R. (1994). Shadows of the Mind. Oxford: Oxford University Press.

Penrose, R. and Hameroff, S. (1995). What 'Gaps'? Reply to Grush and Churchland. Journal of Conscious Studies, 2, 98-112.

(2017). Physics in a minute: The double slit experiment. Plus Magazine, February 5. https://plus.maths.org/content/physics-minute-double-slit-experiment-0. Accessed 3 December 2018.

Place, U. T. (1954). The Concept of Heed. British Journal of Psychology, 45, 243-255.

Place, U. T. (1956). Is Consciousness a Brain Process?. British Journal of Psychology, 47, 44-50.

Pylkkänen, P. (2015). Quantum theory, active information and the mind-matter problem. In E. Zhafarov (Ed.), Contextuality from Quantum Physics to Psychology (pp. 325-334). Advanced series on mathematical psychology, World Scientific.

Rorty, R. (1965). Mind-Body Identity, Privacy, and Categories. The Review of Metaphysics, XIX(1), 24-54.

Rorty, R. (1970). In Defense of Eliminative Materialism. The Review of Metaphysics, XXIV(1), $112-121$.

Ryle, G. (2000). The Concept of Mind. London: Penguin Books.

Smart, J. J. C. (2007). The Mind/Brain Identity Theory. The Stanford Encyclopedia of Philosophy. https://plato.stanford.edu/archives/spr2017/entries/mind-identity/. Accessed 6 March 2019.

Smith, D. W. (2013). Phenomenology. The Stanford Encyclopedia of Philosophy. http://plato.stanford.edu/archives/win2013/entries/phenomenology/. Accessed 25 September 2018.

Strawson, G. (2016). Consciousness Isn't a Mystery. It's Matter. The New York Times, May 16. http://www.nytimes.com/2016/05/16/opinion/consciousness-isnt-a-mystery-its-matter.html?_r=0.

Accessed 4 June 2016.

Wendt, A. (2015). Quantum Mind and Social Science. Cambridge: Cambridge University Press.

Wittgenstein, L. (1953). Philosophical Investigations. G.E.M. Anscombe and R. Rhees (Eds.), G.E.M. Anscombe (Trans.). Oxford: Blackwell. 